\title{
Increased resistin levels in the serum and cerebrospinal fluid of patients with
}

\section{neuromyelitis optica}

Yuan Qi ${ }^{a}$, Kun Jia ${ }^{a}$, Da-Qi Zhang ${ }^{a}$, Ting Li ${ }^{a}$, Li-Min Li ${ }^{a}$, Lin-Jie Zhang ${ }^{a}$, Jing Wang ${ }^{a}$, Chun-Li Gao ${ }^{\text {a }}$,

Li-Sha Sun ${ }^{\mathrm{c}}$, Fu-Dong Shi ${ }^{\text {a, }}{ }^{\text {, }}$ Li Yang ${ }^{\mathrm{a}, *}$

${ }^{a}$ Department of Neurology and Tianjin Neurological Institute, Tianjin Medical University General Hospital,

Tianjin, China.

${ }^{\mathrm{b}}$ Departments of Neurology and Neurosurgery, Barrow Neurological Institute, St. Joseph's Hospital and Medical Center, Phoenix, AZ, USA.

${ }^{\mathrm{c}}$ Department of Clinical Laboratory Center, Tianjin Medical University General Hospital, Tianjin, China.

Corresponding author: Li Yang, M.D., Ph.D. Department of Neurology and Tianjin Neurological Institute,

Tianjin Medical University General Hospital, Tianjin 300052, China, Tel.: +86-22-608-14536. Fax:

+86-22-608-17471, Email: yangli2001@ tmu.edu.cn; yungli2001@yahoo.com 


\begin{abstract}
Introduction: Resistin, which acts as a pro-inflammatory cytokine, has been implicated in the pathogenesis of several autoimmune diseases. However, the involvement of resistin in neuromyelitis optica (NMO), a severe inflammatory central nervous system disorder that targets the optic nerve and spinal cord, remains unclear.

Methods: We measured serum and cerebrospinal fluid (CSF) resistin levels in patients with NMO and controls matched by age and sex. The link between resistin levels and clinical variables in NMO was subsequently assessed.

Results: The concentrations of serum and CSF resistin were significantly higher in patients with NMO than in controls, and decreased following treatment with methylprednisolone. High sensitivity C-reactive protein (hs-CRP) levels and annualized relapse rate positively correlated with resistin levels in patients with NMO.

Conclusion: Resistin might be a useful biomarker of inflammation in NMO, and a potential target for the treatment of NMO.
\end{abstract}

Keywords: resistin; neuromyelitis optica; inflammation; annualized relapse rate; hs-CRP 


\section{Introduction}

Neuromyelitis optica (NMO) is a severe autoimmune inflammatory central nervous system (CNS) disorder that preferentially targets the optic nerve and spinal cord. Identification of the aquaporin 4 (AQP4) antibody, a specific biomarker of NMO pathogenesis, has significantly improved the understanding of NMO [1,2]. The AQP4 antibody specifically combines the foot processes of astrocytes, the antibody-antigen complex activates the complement system, which induces inflammatory responses, granulocyte and macrophage infiltration. In turn, these inflammatory responses produce secondary oligodendrocyte damage, demyelination, and neuronal death.

Resistin is an adipocyte-secreted peptide that induces insulin resistance in mice [3], but is predominantly expressed in monocytes and macrophages in humans [4]. Recent studies have indicated that resistin is able to trigger proinflammatory mechanisms, and is heavily involved in the inflammatory process. Furthermore, resistin is reported to induce the expression of the proinflammatory cytokines tumour necrosis factor $\alpha$ (TNF- $\alpha$ ) and interleukin-6 (IL-6) in vitro [5,6].

Studies have demonstrated that resistin act as an inflammatory mediator in several autoimmune diseases, including rheumatoid arthritis (RA), Sjogren's syndrome (SS), systemic lupus erythematosus (SLE) and myasthenia gravis [5, 7-11]. However, limited studies have investigated the role of resistin in the inflammatory pathogenesis of NMO.

\section{Methods}

\subsection{Subjects}

Patients with NMO $(n=56)$ were recruited between January 2009 and December 2013 from Tianjin Medical University General Hospital. Patients with NMO were diagnosed according to the Wingerchuk 2006 revised criteria [12]. Exclusion criteria included a history of diabetes, cardio- or cerebrovascular diseases, and inflammatory and 
autoimmune diseases other than NMO. All NMO patients were monitored over a 1-y period. In addition, 56 age- and gender- matched healthy controls and 12 other non-inflammatory neurological disorders (ONNDs) as desease controls were collected. ONNDs included 5 amyotrophic lateral sclerosis, 2 hereditary ataxia, and 5 subacute combined degeneration in this study. Informed consent was obtained from all participants prior to the start, and the study was approved by the Tianjin Medical University General Hospital institutional review board and ethics committee.

\subsection{Data and sample collection}

With regard to clinical records, several variables were monitored, including age at onset, disease duration, annualized relapse rate (ARR), glucose levels, triglyceride (TG), total cholesterol (TC), high-density lipoprotein (HDL), low-density lipoprotein (LDL), and high sensitivity C-reactive protein (hs-CRP) levels . The degree of disability in NMO patients was assessed using the Expanded Disability Status Scale (EDSS) score. Serum AQP4 antibody levels were quantified for all NMO patients using CBA-fluorescence immunoprecipitation assays based on transfected HEK293 cells [13].

The 56 serum and 20 CSF samples were obtained within 7 days of an attack, and prior to immunosuppressive treatment for NMO patients. In addition, 56 serum samples from healthy controls and 12 CSF samples from ONNDs were collected. To examine the dynamic changes in resistin levels at different stages of disease, 16 serum samples from NMO patients were collected after at least 4 weeks of treatment. These 16 patients were treated with $500 \mathrm{mg} /$ day of methylprednisolone for the first 5 days, which gradually reduced to a maintenance dose of prednisolone. All serum and CSF samples obtained from participants were stored at $-80{ }^{\circ} \mathrm{C}$ until assay.

\subsection{Measurement of resistin levels}


Serum and CSF resistin concentrations were evaluated using a human resistin enzyme-linked immunosorbent assay (ELISA) kit (eBioscience) according to the manufacturer's instructions. A polyclonal antibody specific for resistin was pre-coated onto a 96-well microplate. Each well was filled with $100 \mu \mathrm{l}$ of resistin standard, diluted serum (1:20) or CSF (1:2) from patients or controls and incubated for $2 \mathrm{~h}$. Next, the samples were incubated with a biotin-labelled secondary polyclonal anti-human resistin antibody. After washing, the streptavidin-horseradish peroxidase (HRP) conjugate was added. Optical densities were measured at $450 \mathrm{~nm}$ using a Microplate reader (SYNERGY2, BioTek). Resistin concentrations were evaluated with reference to a standard curve. The lowest detectable level was $31 \mathrm{pg} / \mathrm{ml}$.

\subsection{Statistical Analysis}

Statistical analysis was performed using SPSS 16.0. Graphs were created using GraphPad PRISM 5. Differences between groups were analysed using a Student's $t$-test when the data were normally distributed. If the data were non-normally distributed, Mann Whitney U tests were used to compare unpaired continuous measures. Categorical variables were assessed using a $\chi^{2}$ test. The differences in resistin levels between the groups (NMO, ONND and healthy controls) were analysed using an analysis of variance (ANOVA). If the ANOVA findings were significant, the effects of glucose, TC, TG, HDL and LDL were investigated by adding these variables to the analysis model as covariates. Differences between NMO subgroups were analysed using a paired $t$-test. The relationships between variables were analysed using Spearman correlation analysis. A P-value of $<0.05$ was considered significant for all statistical analyses.

\section{Results}

Detailed characteristics of the NMO group are displayed in Table 1. As shown in Fig. 
$1 \mathrm{~A}$, the concentrations of serum resistin were significantly higher in patients with NMO than in healthy controls $(7.6 \pm 3.0$ vs $4.1 \pm 1.4 \mathrm{ng} / \mathrm{ml}$; $\mathrm{p}<0.001)$. Furthermore, Fig. 1B demonstrates that serum hs-CRP levels were also significantly increased in the NMO group $(2.0 \pm 1.9 \mathrm{mg} / \mathrm{l}$ vs $0.8 \pm 0.9 \mathrm{mg} / \mathrm{l}, \mathrm{p}=0.017)$. In Fig. $1 \mathrm{C}$, the concentration of CSF resistin was significantly higher in patients with NMO than in patients in the ONND group $(0.25 \pm 0.23 \mathrm{ng} / \mathrm{ml}$ vs $0.04 \pm 0.01 \mathrm{ng} / \mathrm{ml} ; \mathrm{p}=0.001)$

A correlation analysis was performed to investigate a possible relationship between resistin levels, hs-CRP levels, and clinical disease characteristics. Subsequent to this, a significant correlation was observed between resistin and hs-CRP levels in the serum and CSF of NMO patients $(r=0.303, p=0.023$; Fig. 2A; $r=0.622, p=0.003$; Fig. 2B).

A positive correlation was identified between serum resistin levels and the ARR of patients with $\mathrm{NMO}(\mathrm{r}=0.354, \mathrm{p}=0.007$; Fig. 3A $)$, however, no correlations were detected with regard to age at disease onset, disease duration, number of spinal cord segments involved, EDSS score and AQP4 antibody levels.Serum resistin levels were also measured prior to and after treatment in 16 patients with NMO, wherein levels were reduced after treatment $(8.6 \pm 3.3$ vs $4.7 \pm 1.5 \mathrm{ng} / \mathrm{ml}, \mathrm{p}<0.001$; Fig. 3B $)$.

\section{Discussion}

In this case-control study, patients with NMO were found to have higher serum and CSF resistin levels, wherein serum-resistin levels demonstrated a positive correlation with ARR. In addition, the levels of resistin positively correlated with the levels of hs-CRP in the serum and CSF of patients with NMO. These findings suggest that resistin might play an important role in the inflammatory pathogenesis of NMO.

Previous studies have demonstrated increased resistin levels in autoimmune disorders; wherein resistin is reported to significantly contribute to the inflammatory processes 
underlying autoimmune diseases. However, the role of resistin in NMO has not been clearly defined. Resistin was reported to be elevated in both the serum and synovial fluid of RA patients, wherein serum resistin levels correlated with the levels of C-reactive protein, IL-1 $\beta$, erythrocyte sedimentation rate, and disease activity $[8,14,15]$. Accordingly, in the salivary glands of patients with primary SS, increased expression of resistin was found to correlate with lymphocytic inflammation [9]. In addition, an association between serum resistin levels and general inflammation was previously demonstrated in patients with SLE [10]. While NMO is also an inflammatory autoimmune disease with a relatively unknown cause, resistin may play a similar role in the inflammatory mechanisms underlying NMO pathogenesis.

Despite increasing studies concerning the role of resistin in inflammation and related signalling pathways, the exact contribution of resistin to inflammatory pathophysiology remains poorly understood. Studies have demonstrated that the expression of resistin is up-regulated by proinflammatory mediators, including TNF- $\alpha$, IL-1 $\beta$, IL-6, and lipopolysaccharides (LPS) in peripheral blood mononuclear cells (PBMCs) [16]. However, resistin induces the expression of TNF- $\alpha$ and IL- 6 in a manner dependent on the nuclear factor kappa B (NF- $\kappa \mathrm{B})$ signalling pathway [5]. Considering the important roles of IL-6 and TNF- $\alpha$ in the pathogenesis of NMO, resistin might contribute to or modulate the inflammatory processes underlying NMO pathogenesis $[17,18]$. Resistin concentrations in both serum and synovial fluid have already garnered attention as a potential marker of systemic inflammatory disease. However, resistin levels in the CNS have been relatively overlooked. In this study, a marked elevation in CSF resistin levels was observed in patients with NMO compared to those with ONNDs, indicating that CSF resistin might 
also be implicated in the pathogenesis of NMO.

Hs-CRP is a reliable biomarker of inflammation, wherein serum hs-CRP levels are reported to reflect systemic low-grade inflammatory activity. Moreover, hs-CRP has been designated as an inflammatory marker for numerous autoimmune diseases. Studies have confirmed that hs-CRP plays an important role in complement system activation [19], a key step in NMO pathogenesis. Accordingly, serum hs-CRP levels were significantly increased in patients with NMO in the present study. Similar studies have reported that hs-CRP levels (>0.1 mg/l) were significantly higher in patients with NMO at relapse [20], while hs-CRP levels increased with EDSS in NMO patients with extensive brain lesions [21]. Previous studies have also confirmed that hs-CRP is able to induce both the mRNA expression and protein secretion of resistin in PBMCs [22]. In the present study, both serum and CSF resistin levels demonstrated a positive correlation with hs-CRP levels in patients with NMO, indicating that resistin and hs-CRP might interact, or otherwise act co-operatively during NMO pathogenesis.

In the present study, serum-resistin levels decreased after treatment with high dose steroids. Studies indicate that decreased resistin levels reflect successful treatment in patients with RA [23] and inflammatory bowel disease [24]. Current results suggest that resistin might be a potential target for the treatment of NMO, while the reduction of resistin levels might improve the disabling symptoms associated with NMO.

While this was a clinical observation study with a small sample size factoring in only a few inflammatory factors, these findings provide important evidence with regard to the role of resistin in $\mathrm{NMO}$, and indicate that further investigation into the potential mechanisms of resistin in NMO pathogenesis is needed. 


\section{Acknowledgement}

This was supported in part by the National Science Foundation of China; the National Basic Research Program of China; the Tianjin Research Program of Application Foundation and Advanced Technology and the National Key Clinical Specialty Construction Project of China. 


\section{References}

[1] Lennon VA, Wingerchuk DM, Kryzer TJ, et al., A serum autoantibody marker of neuromyelitis optica: distinction from multiple sclerosis, Lancet. 364 (2004) 2106-2112.

[2] Papadopoulos MC, Verkman AS, Aquaporin 4 and neuromyelitis optica, The Lancet Neurology. 11 (2012) 535-544.

[3] Steppan CM, Bailey ST, Bhat S, et al., The hormone resistin links obesity to diabetes, Nature. 409 (2001) 307-312.

[4] Schwartz DR, Lazar MA, Human resistin: found in translation from mouse to man, Trends in endocrinology and metabolism: TEM. 22 (2011) 259-265.

[5] Bokarewa M, Nagaev I, Dahlberg L, et al., Resistin, an adipokine with potent proinflammatory properties, Journal of immunology (Baltimore, Md : 1950). 174 (2005) 5789-5795.

[6] Nagaev I, Bokarewa M, Tarkowski A, et al., Human resistin is a systemic immune-derived proinflammatory cytokine targeting both leukocytes and adipocytes, PloS one. 1 (2006) e31.

[7] Gheita TA, El G, II, El Shazly RI, et al., Elevated serum resistin in juvenile idiopathic arthritis: relation to categories and disease activity, Journal of clinical immunology. 33 (2013) 297-301.

[8] Forsblad d'Elia H, Pullerits R, Carlsten H, et al., Resistin in serum is associated with higher levels of IL-1Ra in post-menopausal women with rheumatoid arthritis, Rheumatology (Oxford, England). 47 (2008) 1082-1087.

[9] Bostrom EA, d'Elia HF, Dahlgren U, et al., Salivary resistin reflects local inflammation in Sjogren's syndrome, The Journal of rheumatology. 35 (2008) 2005-2011. 
[10]Almehed K, d'Elia HF, Bokarewa M, et al., Role of resistin as a marker of inflammation in systemic lupus erythematosus, Arthritis research \& therapy. 10 (2008) R15.

[11]Zhang DQ, Wang R, Li T, et al., Remarkably increased resistin levels in anti-AChR antibody-positive myasthenia gravis, Journal of neuroimmunology. 283 (2015) 7-10. [12]Wingerchuk DM, Lennon VA, Pittock SJ, et al., Revised diagnostic criteria for neuromyelitis optica, Neurology. 66 (2006) 1485-1489.

[13] Wang J, Li X, Zhang DQ, et al., Quantitative analysis of aquaporin-4 antibody in longitudinally extensive transverse myelitis, Journal of neuroimmunology. 278 (2015) 26-29.

[14]Molenaar ET, Voskuyl AE, Familian A, et al., Complement activation in patients with rheumatoid arthritis mediated in part by C-reactive protein, Arthritis and rheumatism. 44 (2001) 997-1002.

[15] Schaffler A, Ehling A, Neumann E, et al., Adipocytokines in synovial fluid, Jama. 290 (2003) 1709-1710.

[16] Kaser S, Kaser A, Sandhofer A, et al., Resistin messenger-RNA expression is increased by proinflammatory cytokines in vitro, Biochemical and biophysical research communications. 309 (2003) 286-290.

[17]Chang KH, Ro LS, Lyu RK, et al., Biomarkers for neuromyelitis optica, Clinica chimica acta. 440 (2015) 64-71.

[18]Uzawa A, Mori M, Sawai S, et al., Cerebrospinal fluid interleukin-6 and glial fibrillary acidic protein levels are increased during initial neuromyelitis optica attacks, Clinica chimica acta. 421 (2013) 181-183. 
[19]Thompson D, Pepys MB, Wood SP, The physiological structure of human C-reactive protein and its complex with phosphocholine, Structure (London, England : 1993). 7 (1999) 169-177.

[20]Doi H, Matsushita T, Isobe N, et al., Hypercomplementemia at relapse in patients with anti-aquaporin-4 antibody, Multiple sclerosis (Houndmills, Basingstoke, England). 15 (2009) 304-310.

[21]Cheng C, Jiang Y, Chen X, et al., Clinical, radiographic characteristics and immunomodulating changes in neuromyelitis optica with extensive brain lesions, BMC neurology. 13(2013) 72.

[22] Hu WL, Qiao SB, Li JJ, Decreased C-reactive protein-induced resistin production in human monocytes by simvastatin, Cytokine. 40 (2007) 201-206.

[23] Gonzalez-Gay MA, Garcia-Unzueta MT, Gonzalez-Juanatey C, et al., Anti-TNF-alpha therapy modulates resistin in patients with rheumatoid arthritis, Clinical and experimental rheumatology. 26 (2008) 311-316.

[24]Karmiris K, Koutroubakis IE, Xidakis C, et al., The effect of infliximab on circulating levels of leptin, adiponectin and resistin in patients with inflammatory bowel disease, European journal of gastroenterology \& hepatology. 19 (2007) 789-794. 


\section{Fig. legend}

Fig. 1 Levels of serum resistin, hs-CRP, and CSF resistin in patients with NMO. (A) Serum resistin levels in 56 patients with NMO and 56 healthy controls. (B) Serum hs-CRP levels in 56 patients with NMO and 56 healthy controls. (C) Concentration of CSF resistin in 20 patients with NMO and 12 patients with ONNDs.

Abbreviations: hs-CRP, high sensitivity C-reactive protein; CSF, cerebrospinal fluid; NMO, neuromyelitis optica; ONNDs, other non-inflammatory neurological disorders.

Fig. 2 Correlation between resistin and hs-CRP levels in the serum and CSF of patients with NMO. (A) Relationship between serum resistin and hs-CRP levels in 56 patients with NMO. (B) Relationship between CSF resistin and hs-CRP levels in 20 patients with NMO. Abbreviations: hs-CRP, high sensitivity C-reactive protein; CSF, cerebrospinal fluid; NMO, neuromyelitis optica.

Fig. 3 Associations of serum resistin levels with the clinical features of the NMO. (A) Relationship between serum resistin levels and ARR in 56 patients with NMO. (B) Variation in serum resistin levels with regard to the high dose MP therapy in 16 patients. Abbreviations: NMO, neuromyelitis optica; ARR, annualized relapse rate; MP, methylprednisolone. 


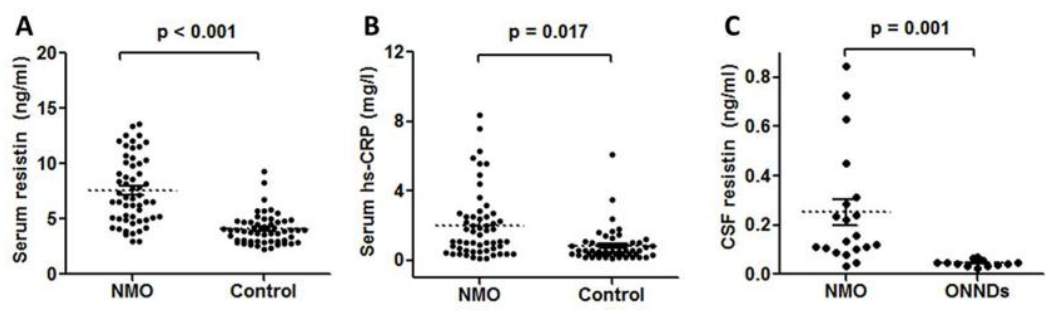




A

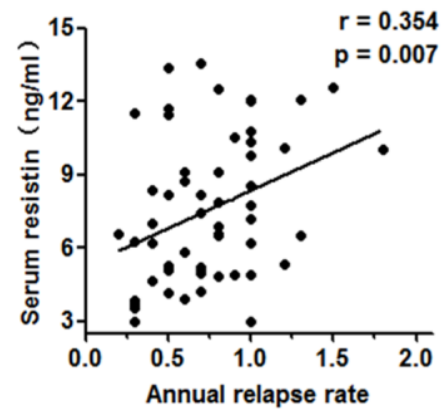

B

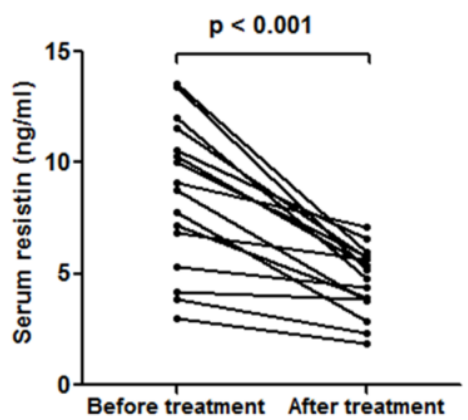


Table 1 Demographic and clinical features of patients with NMO and healthy control subjects

\begin{tabular}{llll}
\hline & Patients with NMO & Health Controls & P-value \\
\hline Gender, female/male & $47 / 9$ & $47 / 9$ & NS \\
Age (y) & $50.9 \pm 13.1$ & $48.2 \pm 12.2$ & NS \\
Onset age (y) & $43.6 \pm 13.2$ & - & - \\
Disease duration (y) & $7.0 \pm 6.0$ & - & - \\
Annualized relapse rate & $0.7 \pm 0.3$ & - & - \\
Number of segments of spinal cord involved & $6(3-18)$ & - & - \\
Expanded Disability Status Scale score & $5(2.0-8.5)$ & - & - \\
AQP4 antibody positivity & $44(78.6 \%)$ & - & NS \\
BMI(kg/m $\left.{ }^{2}\right)$ & $23.3 \pm 1.9$ & $23.1 \pm 1.7$ & $<0.001$ \\
Glucose (mmol/l) & $5.3 \pm 0.8$ & $4.5 \pm 0.5$ & $<0.001$ \\
TC (mmol/l) & $5.0 \pm 1.1$ & $4.2 \pm 0.5$ & NS \\
TG (mmol/l) & $1.2 \pm 0.7$ & $1.0 \pm 0.3$ & 0.025 \\
HDL (mmol/l) & $1.3 \pm 0.4$ & $1.5 \pm 0.4$ & $<0.001$ \\
LDL (mmol/l) & $3.1 \pm 0.9$ & $2.3 \pm 0.6$ & \\
\hline Data are expres & &
\end{tabular}

Data are expressed as means \pm SD unless otherwise indicated; NMO = neuromyelitis optica; 